\title{
Cutaneous Plasmacytoma: An Uncommon Entity
}

Sanober Nusrat ${ }^{\star}$, Aamina T Shakir and Amany R Keruakous

Department of Internal Medicine, University of Oklahoma Health Sciences Center, Oklahoma City, Oklahoma, USA

*Corresponding author: Sanober Nusrat, Department of Internal Medicine, University of Oklahoma Health Sciences Center, 800 Stanton L Young Blvd, AAT 6300, Oklahoma City, Oklahoma, USA, Tel: + 516-324-6510; E-mail: Sanober-nusrat@ouhsc.edu

Rec date: October 05, 2018; Acc date: October 06, 2018; Pub date: October 08, 2018

Copyright: (c) 2018 Nusrat S, et al. This is an open-access article distributed under the terms of the creative common's attribution license, which permits unrestricted use, distribution, and reproduction in any medium, provided the original author and source are credited.

Citation: Nusrat S, Shakir AT, Keruakous AR (2018) Cutaneous Plasmacytoma: An Uncommon Entity. J Cancer Sci Ther 10: 293. doi:10.4172/1948-5956.1000i101

\section{Clinical Image}

Extramedullary plasmacytoma is a manifestation of multiple myeloma in which a discrete mass of neoplastic monoclonal plasma cells forms within soft tissue [1]. Cutaneous plasmacytoma remains an uncommon entity. We present a case of a 61 -year-old female with a seven-year history of multiple myeloma diagnosed in the setting of a pathological fracture secondary to multiple lytic lesions. Patient initially went into remission after receiving Bortezomib-based chemotherapy followed by autologous stem cell transplant. Following transplant, she was noted to be in CR2 with no evidence of light chain disease for 3 years. She then developed an enlarging anterior abdominal wall mass which was consistent with plasmacytoma [2]. Core biopsy of the lesion showed CD138 positive and kappa-restricted plasma cells with a high proliferation index, thereby establishing disease relapse. The lesions continued to progress in size and number and she was hospitalized for concerns of superimposed infection. Vitals on admission were within normal limits. On exam, multiple foulsmelling, fungating tumors occupying a dimeter of 5 centimeters or more were noted on the infraumbilical region of the abdominal wall (Figures 1 and 2).

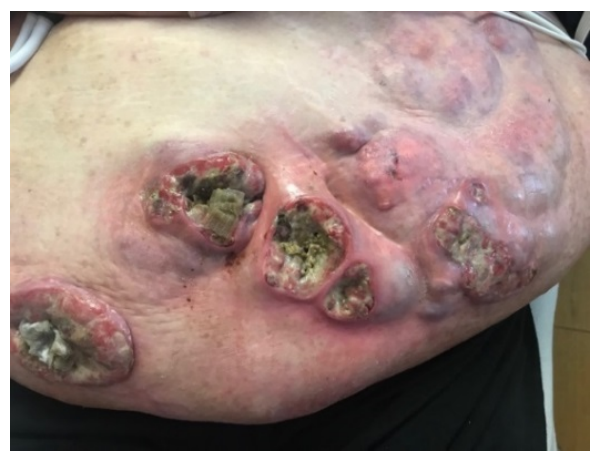

Figure 1: Multiple foul-smelling, fungating tumors.

There was scant purulence but no surrounding erythema Laboratory results on admission were only significant for low hemoglobin, also at baseline. White cell count and infectious markers were unremarkable [3]. Computerized tomography imaging showed extensive burden of subcutaneous tumor without evidence of soft tissue fluid collection or gas (Figure 3). Dermal lesions were not thought to be infected, so antimicrobial therapy was not instituted. No surgical intervention was deemed necessary and wound care was recommended. To conclude, it is important to emphasize that multiple myeloma can relapse with extramedullary subcutaneous plasmacytoma. While it is important to rule out superimposed infection or associated abscess, they often do not require antimicrobials or debridement. Radiotherapy can be an important treatment modality in such instances.

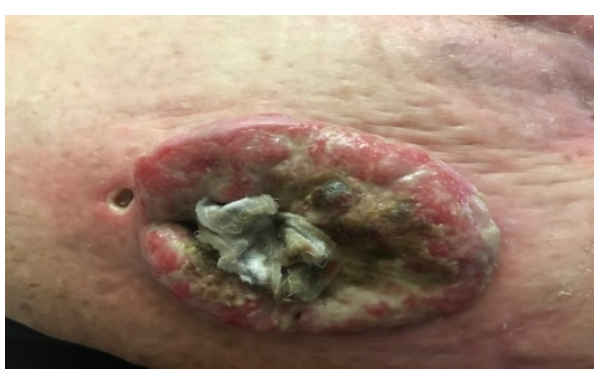

Figure 2: Fungating tumors on the infraumbilical region of the abdominal wall.

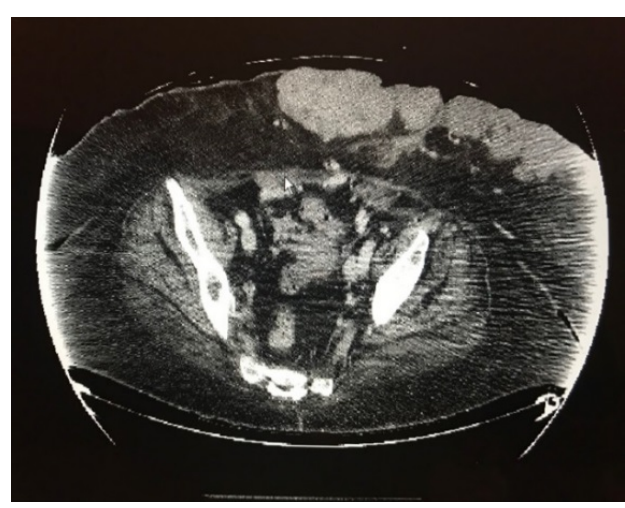

Figure 3: Computerized tomography imaging showed extensive burden of subcutaneous tumor.

\section{References}

1. Ge S, Zhu G, Yi Y (2018) Extramedullary plasmacytoma of the larynx: Literature review and report of a case who subsequently developed acute myeloid leukemia. Oncol Lett 16: 2995-3004.

2. Wazzan T, Kashtwari D, Almaden WF, Gong Y, Chen Y, et al. (2018) Radiographic bone loss and the risk of Medication-Related Osteonecrosis of the Jaw (MRONJ) in multiple myeloma patients: A retrospective case control study. Spec Care Dentist.

3. Goel A, Insa R, Gaur MK, Garg PK (2018) Palliative surgery for metastatic fungating phyllodes tumors: A series of two cases. Perm J 19: 22. 